\title{
Skin care products: friend or foe
}

\begin{abstract}
Skin, the largest organ of human integumentary system, which interacts with the environment and protect the body from various pathogens and toxic chemicals, that comes in contact directly or indirectly in daily life through various sources like skin care products available in markets. In this paper we review a list of chemicals which are known to cure skin problems but in turn lead to severe acute diseases. Sodium lauryl sulphate, diethanolamine, nanoparticles (zinc oxide, titanium oxide), monoethanolamine, triethanolamine, propylene glycol, butylene glycol, are found in most of the skin care products which have both beneficial and toxic effects. This study gives a clear picture of the research done on the role of the ingredients and its effects on and inside the human skin.
\end{abstract}

Keywords: skin, market, sodium lauryl sulphate, diethanolamine, nanoparticle, monoethanolamine, triethanolamine, propylene glycol, butylenes glycol, skin care products
Volume 2 Issue $6-2018$

\section{Atefa Noorain}

AET Laboratories Private Limited, Hyderabad, India

Correspondence: Atefa Noorain, AET Laboratories Private Limited, Hyderabad, India, Tel +9198 8569 469I Email atefanuri@gmail.com

Received: August 15, 2018 | Published: December 31, 2018

\section{Introduction}

Human skin is the largest organ of the body, which is often taken for granted. An adult's skin comprises between 15 and 20 percent of the total body weight. Each square centimeter has 6 million cells, 5,000 sensory points, 100 sweat glands and 15 sebaceous glands. Skin is the most sensitive part of human body. According to the researchers everything we apply on skin pass through the layer stratum corneum. Most of the people use variety of cosmetics, makeup things, such as Moisturizers, Lipsticks, Lotions, Face creams, Shampoos, Shaving Creams, Deodorants, Hair Dyes and etc., Some people who are sensitive to those things are affected in varied levels. The Allergens present in them are the main cause for the allergy. Phthalates, Acrylamine, Formaldehyde and Ethylene Oxide are some chemicals present in the cosmetics act as Allergens. They are the things which cause an allergic reaction in human body. It is no doubt to say that beauty means good health. But some of the harmful synthetic cosmetics containing various chemicals affect the body health and the beauty of many persons who are hypersensitive to those things. The aim of the study is to differentiate between the useful \& toxic chemicals present in various skin care products $\&$ how the human skin reacts to those chemicals when comes in contact. ${ }^{1-5}$

\section{Materials \& methods}

\section{Sodium lauryl sulphate (SLS) ${ }^{6-8}$}

a. It is found in cleansers that foam, car washes, engine degreasers and baby shampoos

b. It is highly toxic synthetic substance

c. It can lead to health issues ranging from eczema to canker sores

d. It causes inflammation of skin \& can weaken immune system

e. Causes intestinal toxicity, urinary tract infection, bladder \& kidney infection, genital disorder, eye irritation, skin rashes, hair loss \& allergic reactions

f. It is used to violate the skin's natural barrier function in lab test

g. It causes the body to have stronger reactions to other irritants including allergens

h. Products containing SLS: Shampoo, bubble bath, bar soap, dish soap, tooth paste \& mouth wash i. SLS chemistry: SLS exert its effect on proteins by forming a chemical bridge between the fat soluble $\&$ water soluble parts of protein molecule. This disrupts the hydrophobic forces needed to maintain protein structure $\&$ the molecule collapses, rendering it useless. This effect is usually reversible

a) The result of this is 2-fold, firstly existing proteins are damaged leading to an increase in the amount of healing required by body. Secondly new proteins can be damaged \& cell disrupted while they are under construction. It is exactly this type of activity that can lead to early stages of skin cancer

b) The process can be severe, that skin layers may separate \& inflame due to its protein denaturing property

c) Carcinogenic nitrates can form in the manufacturing of SLS or by inter-reaction with other nitrogen bearing ingredients within a formulation utilizing this ingredient

d) A single shampooing can produce more cancer-causing nitrates in the body than eating a pound of bacon, which is very high in nitrates

j. One study showed $41.8 \%$ of 160 people showed irritant reaction after exposure to $0.5 \%$ SLS $^{9}$

\section{Diethanolamine(DEA) $)^{10,11}$}

i. It is used as wetting agent in shampoos \& keeps a favorable consistency in lotions \& creams

ii. It has been linked with kidney, liver \& other organ damage according to research done

iii. One study found that topical application of DEA in rodents resulted in anemia, kidney degeneration \& nerve damage to the brain \& spinal cord

iv. It readily absorbed through skin \& has been linked with stomach, esophagus, liver \& bladder cancer

v. 200 million pounds of DEA are produced annually in U.S most of which goes to personal care products

vi. DEA chemistry: It can react with other ingredient in cosmetic formula to form an extremely potent carcinogen called NDEA (nitrosodiethanol amine) 
vii. Cosmetic ingredients contaminated with DEA:
a. Cocamide DEA
b. DEA lauryl sulphate
c. Lauramide DEA
d. Linoleamide DEA
e. Oleamide DEA

\section{Monoethanol amine (MEA) $)^{12-15}$}

a) It is a corrosive liquid, harmful if swallowed

b) It can cause skin burns \& can lead to corneal burns with possible permanent damage \& CNS depression

c) Ingestion may result in irritation of mouth, respiratory tract \& digestive tract $\&$ may cause drowsiness $\&$ dizziness

d) It is a potential skin sensitizer

e) Inhalation may cause asthma like symptoms

\section{Triethanol amine(TEA) ${ }^{16}$}

a) TEA is an amine, an organic compound derived from ammonia which is used in low concentration as an alkalizing agent to increase the $\mathrm{pH}$ of cosmetic formulations

b) It is a possible human carcinogen

c) It is used to adjust the $\mathrm{pH}$ balance, but toxic \& causes eye problems, \& dryness of skin \& hair

d) TEA in sunscreen occasionally causes contact allergy like dermatitis

e) TEA causes an increased incidence of tumor growth in the liver via a choline-depletion mode of action \& that this effect is likely caused by inhibition of choline uptake by cells in female B6C3F 1 mice, but not in Fischer 344 rats

f) Products containing TEA: eye liner, mascara, eye shadow, blushers, make-up bases \& foundations, as well as fragrances, hair care products, wave sets, shaving products, sunscreen \& skin cleansing products

\section{Propylene glycol (PG) ${ }^{11-18}$}

a. It is petroleum by product \& is a synthetic ingredient used as a humectants which causes retention of moisture content of skin or cosmetic products by preventing the escape of moisture \& water

b. It is used as an industrial anti-freeze to de-ice airplanes

c. It easily penetrate skin can weaken protein \& cellular structure.

d. PG is a solvent that it dissolves through a stainless steel tank in 48 hours

e. PG is so toxic that it requires workers to wear protective gloves, clothing \& goggles

f. It is an immune toxicant causes many allergic reactions \& can cause liver abnormality \& kidney damage.

g. It is found to cause skeletal muscular damage in rats \& rabbit

h. It is a known irritant \& sensitizer causing dryness, erythema \& even blistering i. It directly alters cell membrane to cause thickening of skin, skin dehydration \& chronic damage to skin

j. Ptach test helps to diagnose the toxicity of PG

k. PG chemistry: It is a chemical made by reaction of propylene oxide with water

a. It is chemically neutral

b. It is cosmetic form of mineral oil found in automatic brake \& hydraulic fluid

1. Products containing PG: shampoo, deodorant, detergent, styling mousse, mascara, soap, skin cream, baby powder, conditioner toner, after shave, baby wipes

a. It is also found in tyre sealant rubber cleaner, de-icer, stain remover, fabric softner, degreaser, paints, adhesive

\section{Butylene glycol (BTG) ${ }^{19-25}$}

i. It is used as solvents \& viscosity decreasing agents in cosmetics $\&$ personal care products

ii. It is been proven to be gentle \& non-irritating to the skin, even in higher concentrations

iii. BTG chemistry: It is an organic molecule with 2 alcohol groups, used in cosmetics as a humectant to bind moisture $\&$ hold water to the skin

iv. Products containing BTG: shampoo, conditioner, moisturizer, foundation, cleanser, eye cream \& sunscreen

\section{Titanium dioxide $\left(\mathrm{TIO}_{2}\right)^{26-29}$}

a. It is a common ingredient in sun protection creams

b. It is a physical sunscreen that reflects UV light before it can damage the skin

c. It reduces risk of skin cancer

d. It does not penetrate normal skin

e. If skin is lacerated or otherwise exposed, micronized $\mathrm{TiO}_{2}$ can cause detrimental cellular effects.

f. It can be a mild skin irritant in some people.

g. It is listed as a safe pigment in skin care products

\section{Conclusion}

Thus in the summary of the paper we get a clear picture of how different chemical can act as Friend or Foe to the human body. The 2 chemicals Titanium dioxide \& Butylene glycol are proved to be helpful to the human skin without any severe side effects. Hence in future the detailed composition of the skin products need to be checked before commencement of its use. The future research can be done taking particular skin products and testing its quality and beneficial effects on human body.

\section{Acknowledgments}

None.

\section{Conflicts of interest}

Author declares that there is no conflicts of interest. 


\section{References}

1. http://www.preventcancer.com/consumers/cosmetics/ diethanolamine.htm

2. http://www.preventcancer.com/consumers/cosmetics/ diethanolamine.htm

3. http://www.preventcancer.com/consumers/cosmetics/ diethanolamine.htm

4. http://www.apcoindustries.com/Monoethanolamine.pdf

5. http://www.natural-health-information-centre.com/propylene-glycol. html

6. http://antiagingchoices.com/harmful_ingredients/propylene_glycol. htm

7. http://www.propylene-glycol.com/what_is.html

8. http://www.propylene-glycol.com/cosmetics.html

9. http://www.health-report.co.uk/triclosan.html

10. http://www.naturalnews.com/triclosan.html

11. http://www.beyondpesticides.org/pesticides/factsheets/ Triclosan\%20cited.pdf

12. http://en.wikipedia.org/wiki/Triethanolamine\#Allergic_reactions

13. http://www.cosmeticsinfo.org/ingredient_details.php?ingredient id $=622$

14. http://www.lotioncrafter.com/triethanolamine-nf.html
15. http://www.natural-health-information-centre.com/propylene-glycol. html

16. http://antiagingchoices.com/harmful_ingredients/propylene_glycol. htm

17. http://www.cosmeticsinfo.org/ingredient_details.php?ingredient_ $\mathrm{id}=477$

18. http://www.nuskin.com/en_BE/corporate/company/science/hot topics/butylene_and_propyleneglycol.html

19. http://truthinaging.com/ingredients/butyline-glycol

20. http://www.livestrong.com/article/277187-health-effects-of-usingtitanium-oxide-in-skin-cream/

21. http://www.organicmakeup.ca/ca/titaniumdioxide.asp

22. http://truthinaging.com/ingredients/titanium-dioxide

23. http://www.herballuxuries.com/human-skin.html

24. http://en.wikipedia.org/wiki/Human_skin\#Nanoparticles

25. http://www.squidoo.com/sls

26. http://www.natural-health-information-centre.com/sls-healthimplications.html

27. http://www.natural-skincare-authority.com/cosmetic-chemicals.html

28. http://www.selfgrowth.com/articles/Maldonado1.html

29. http://www.organichealthandbeauty.com/10-Most-CommonChemicals ep 55-1.html 